\title{
Qualidade de vida de idosos dos centros-dia do Regado e São Tomé - Portugal
}

Recebido em: 08/12/2010

Aceito em: 26/07/2011

\author{
Pâmela Araujo Cimirro' \\ Renata Rigon ${ }^{2}$ \\ Margarida Maria da Silva Vieira ${ }^{3}$ \\ Helena Maria Carvalhinha Teles de \\ Castro Gonçalves Pereira ${ }^{4}$ \\ Marion Creutzberg 5
}

Este estudo objetivou avaliar a qualidade de vida de idosos frequentadores dos centros-dia do Regado e São Tomé de Portugal, através da aplicação dos instrumentos Whoqol-Bref e Whoqol-Old numa abordagem quantitativa. A amostra foi constituída de 30 idosos. A média de idade foi de 78,23 anos. Os escores médios dos domínios Social, Funcionamento do Sensório e Autonomia foram os mais elevados. Estar na companhia de idosos em centros-dia parece ter influência positiva nas relações sociais. A perda sensorial não impacta negativamente, o que expressa avanços na inclusão e no acesso às tecnologias assistivas. Descritores: Idoso, Qualidade de Vida, Centros de Convivência e Lazer, Envelhecimento.

\section{Quality of life of elderly people from Regado and Sao Tome - Portugal Day-Centres}

The aim of this study was to evaluate the quality of life of elderly members from the Sao Tome and Regado Centers-Day from Portugal, by means of application of the Whoqol-Bref and Whoqol-Old in a quantitative approach. The average age was 78,23 years. The mean scores of Social Relationships domain, Sensory Abilities domain and Autonomy were the highest. The possibility of being in the company of elderly at the Centers-Day seems to have positive influence on social relations. The loss of sensory function does not impact negatively, which reflects advances in inclusion and access to assistive technologies.

Descriptors: Aged people, Quality of Life, Centers of Connivance and Leisure, Aging.

\section{Calidad de vida de los ancianos en Centros-Dia del Regado y São Tomé - Portugal}

Este estudio tuvo como objetivo evaluar la calidad de vida de los ancianos de los Centros-Día de Regado y de Sán Tomé - Portugal, por la aplicación del Whoqol-Bref y Whoqol-Old con un enfoque cuantitativo. La muestra consistió de 30 ancianos. La edad promedia fue de 78,23 años. Las puntuaciones medias de los dominios Social, Funcionamiento del Sensorial y la Autonomía eran los más altos. Estar en la compañía de los ancianos en Centros-Día, parece tener influencia positiva sobre las relaciones sociales. La pérdida sensorial no afecta negativamente, lo que refleja los avances en la inclusión y el acceso a tecnologías de asistencia.

Descriptores: Anciano, Calidad de Vida, Centros de Ocio y Convivencia, Envejecimiento.

\section{INTRODUÇÃO}

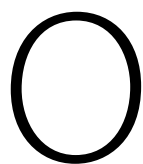
número de pessoas idosas residentes em Portugal duplicou nos últimos 40 anos, provocando o alargamento do topo da pirâmide etária. Em 2009, o Instituto Nacional de Estatística identificou 10.637.713 habitantes residentes em Portugal. Desses, 17,9\% têm 65 anos ou mais. A expectativa de vida é de 81,8 anos para mulheres e 75,8 para homens ${ }^{(1)}$.

O envelhecimento é uma fase natural da vida, determinada basicamente por três fatores: genética, estilo de vida e ambiente. Nesse momento, há um início progressivo de uma deterioração das funções vitais do corpo e ocorrem também alterações da função imunológica, levando a um provável aumento do risco para desenvolver doenças infecciosas, autoimunes ou malignas.
O paradigma do envelhecimento bem-sucedido vê o sujeito como pró-ativo, acumulando recursos que são úteis na adaptação à mudança e que estão ativamente envolvidos na manutenção do bem-estar, acompanhado de qualidade de vida (QV). Os modelos de QV vão desde a satisfação com a vida até modelos baseados em conceitos de independência, controle, competências sociais e cognitivas. Para a Organização Mundial de Saúde (OMS), QV"é a percepção do indivíduo de sua posição na vida, no contexto da cultura e sistemas de valores nos quais vive e em relação a seus objetivos, expectativas e preocupações"(2).

É importante entender como o próprio idoso percebe sua qualidade de vida. Estudos demonstram que existe

1 Enfermeira graduada pela Pontifícia Universidade Católica do Rio Grande do Sul - PUCRS.

2 Enfermeira graduada pela Pontifícia Universidade Católica do Rio Grande do Sul - PUCRS.

3 Enfermeira. PhD, Doutora em Filosofia, Instituto de Ciências da Saúde - Porto, Universidade Católica Portuguesa.

4 Enfermeira. Mestre em Ciências de Enfermagem. Instituto de Ciências da Saúde - Porto, Universidade Católica Portuguesa.

5 Enfermeira. Doutora em Gerontologia Biomédica. Faculdade de Enfermagem, Nutrição e Fisioterapia da PUCRS. E-mail: marionc@pucrs.br 
uma tendência de piores avaliações por parte do cuidador do que pelo próprio idoso(3), talvez pelo fato de os próprios minimizarem seu estado ou pelo fato de os cuidadores enxergarem com maior fragilidade a situação dos idosos.

Para que ações de saúde, individuais e coletivas, possam contribuir para uma melhor QV na velhice, é fundamental que se conheçam as reais necessidades, os problemas e as preocupações que as pessoas enfrentam. Assim, a avaliação da QV é determinante no processo de cuidar em enfermagem, no qual são considerados os aspectos biopsicossociais e espirituais vivenciados pelo idoso e pela família. O cuidado prevê a integração de todas as dimensões do viver da pessoa idosa, favorecendo a exaltação da vida no processo de envelhecer, na utilização de seus potenciais, de suas capacidades, dos recursos, das condições de saúde, evoluindo para um contínuo desenvolvimento pessoal ${ }^{(4)}$.

\section{OBJETIVO}

Avaliar a qualidade de vida dos idosos frequentadores de centros-dia da cidade do Porto, Portugal.

\section{MÉTODO}

Estudo descritivo transversal, com abordagem quantitativa. Embora o desenho transversal não seja muito utilizado para estudos sobre a qualidade de vida, o método é considerado relevante quando seu objetivo é obter um perfil ou dados de base de um grupo específico ou quando os resultados são comparados aos dados de outros grupos ${ }^{(5-6)}$.

$O$ estudo foi realizado com idosos frequentadores de centros-dia da cidade do Porto - Portugal. Os centros-dia estão preparados para prestar apoio à saúde e medicamentoso, oferecer atividades de lazer e apoio domiciliar aos idosos residentes nas áreas adstritas. À noite fecham, não servindo como moradia. $\mathrm{O}$ atendimento tem um custo e é coberto, em parte, pelo sistema público de saúde e, em outra, pelos próprios idosos $^{(7)}$. Foram entrevistados 30 idosos com mais de 60 anos, lúcidos, orientados, usuários dos centros-dia do Regado e São Tomé. Embora na Europa se considere idoso, para fins de políticas públicas, a pessoa a partir de 65 anos, seguiu-se o critério do Brasil, para que se possa realizar, oportunamente, comparações entre grupos. Além disso, os centros-dia na cidade do Porto aceitam pessoas a partir dos 60 anos.

A coleta foi realizada por meio de entrevistas, com o instrumento genérico de qualidade de vida, Whoqol-Bref ${ }^{(8)}$, e o instrumento específico para a avaliação da qualidade de vida em idosos, Whoqol-Old ${ }^{(9)}$, da Organização Mundial de Saúde. Além da aplicação do instrumento, foram coletados os dados de identificação referentes a sexo, idade, profissão e escolaridade. Como o percentual de idosos com dificuldades de leitura era elevado, o instrumento foi aplicado por entrevistador(a), minimizando erros advindos da leitura e compreensão do instrumento ${ }^{(10)}$.

Os dados quantitativos oriundos da aplicação do WhoqolBref e Whoqol-Old foram organizados em um banco de dados do SPSS 12.0. Foram analisados por estatística descritiva com cálculo da frequência média, desvio-padrão e análise inferencial com intervalo de confiança de $95 \%$, utilizando testes estatísticos (qui-quadrado, Teste T-Student, Anova, correlação de Pearson), sendo considerado estatisticamente significante o p-value inferior a 0,05(6).

A partir das sintaxes oferecidas pelo Whoqol Group para o Whoqol-Bref e o Whoqol-Old, os resultados dos escores dos domínios podem ser apresentados de 4-20 ou de 0-100, e ambos foram utilizados para fins de discussão com outros estudos.

O estudo integra o projeto aprovado pelo Comitê de Ética e Pesquisa da PUCRS, sob parecer 568/06, e obteve autorização da Universidade Católica Portuguesa - Portugal e pelos centrosdia do Regado e São Tomé. Os idosos assinaram um Termo de Consentimento Livre e Esclarecido aceitando participar do estudo e autorizando a utilização dos dados, mantendo seu anonimato. Os analfabetos consentiram verbalmente, sendo feito o registro no termo.

\section{RESULTADOS}

Do estudo, participaram $70 \%$ mulheres (21) e $30 \%$ homens (9). A idade média foi de 78,23 $( \pm 7,8)$. Quanto à escolaridade, a média dos anos de estudo foi de $3,73( \pm 3,1)$

Tabela 1 - Distribuição dos escores segundo os domínios do Whoqol-Bref $(n=30)$

\begin{tabular}{|c|c|c|c|c|c|c|}
\hline \multirow[b]{2}{*}{ Domínios } & \multicolumn{3}{|c|}{ Sintaxe 4-20 } & \multicolumn{3}{|c|}{ Sintaxe 0-100 } \\
\hline & Mínimo & Máximo & Média (dp) & Mínimo & Máximo & Média (dp) \\
\hline Físico & 8,00 & 16,00 & $12,55( \pm 2,07)$ & 10,71 & 96,43 & $51,30( \pm 22,42)$ \\
\hline Psicológico & 8,67 & 16,67 & $12,93( \pm 2,04)$ & 20,83 & 91,67 & $60,27( \pm 18,26)$ \\
\hline Social & 6,67 & 18,67 & $13,95( \pm 3,04)$ & 16,67 & 91,67 & $62,22( \pm 19,04)$ \\
\hline Ambiental & 8,00 & 16,50 & $13,73( \pm 1,89)$ & 25,00 & 78,13 & $60,83( \pm 11,85)$ \\
\hline QV Global & 4,00 & 16,00 & $10,80( \pm 3,46)$ & - & - & - \\
\hline QV Global (1-5) & 1,00 & 4,00 & $2,70( \pm 0,86)$ & - & - & - \\
\hline QV geral (q1) & - & - & - & 0,00 & 100 & $51,66( \pm 21,70)$ \\
\hline Saúde geral (q2) & - & - & - & 0,00 & 100 & $33,33( \pm 32,38)$ \\
\hline
\end{tabular}


Tabela 2 - Distribuição dos escores segundo os domínios do Whoqol-Old ( $\mathrm{n}=30)$

\begin{tabular}{|l|c|l|l|l|l|l|}
\hline & \multicolumn{3}{|c|}{ Sintaxe 4-20 } & \multicolumn{3}{c|}{ Sintaxe 0-100 } \\
\hline Domínios & Mínimo & Máximo & Média (dp) & Mínimo & Máximo & Média (dp) \\
\hline Funcionamento do sensório & 6,00 & 20,00 & $14,53( \pm 4,40)$ & 12,50 & 100,00 & $65,83( \pm 27,50)$ \\
\hline Autonomia & 8,00 & 20,00 & $14,53( \pm 3,38)$ & 25,00 & 100,00 & $65,83( \pm 21,13)$ \\
\hline Atividades passadas, presentes e futuras & 8,00 & 18,00 & $13,00( \pm 2,47)$ & 25,00 & 87,50 & $56,25( \pm 15,48)$ \\
\hline Participação social & 10,00 & 19,00 & $13,83( \pm 2,42)$ & 37,50 & 93,75 & $61,45( \pm 15,13)$ \\
\hline Morte e morrer & 4,00 & 20,00 & $14,33( \pm 4,45)$ & 0,00 & 100,00 & $64,58( \pm 27,87)$ \\
\hline Intimidade & 7,00 & 20,00 & $14,13( \pm 4,02)$ & 18,75 & 100,00 & $63,33( \pm 25,14)$ \\
\hline QV Global-Old & 10,17 & 18,33 & $14,06( \pm 1,70)$ & 38,54 & 89,58 & $62,88( \pm 10,64)$ \\
\hline
\end{tabular}

e $80 \%$ dos idosos estudaram até quatro anos. No que diz respeito às profissões, $36,7 \%$ atuaram no comércio e $26,7 \%$, nos serviços domésticos ${ }^{(11)}$.

Na avaliação da QV, por meio do Whoqol-Bref (tabela 1), o domínio com maior correlação positiva com a QV Global foi o físico, ou seja, à medida que aumenta ou diminui a satisfação com esse aspecto, aumenta ou diminui a qualidade de vida $(r=0,658)$.

O escore médio obtido na QV Global foi de 9,61 $( \pm 3,07)$ para mulheres e 13,55 $( \pm 2,78)$ para homens, sendo essa diferença significativa $(p=0,003)$. A diferença também foi significativa nos domínios físico, psicológico e ambiental e também na avaliação geral da saúde, sempre com escores superiores para os homens. Não houve diferença estatisticamente significativa entre faixa etária e QV.

No domínio Funcionamento do sensório, a faceta de maior impacto foi a que se refere à influência da perda dos sentidos na participação de atividades $(r$ $=0,847$ ). Na Autonomia, foi a liberdade em tomar decisões que demonstrou maior influência $(r=0,902)$. No domínio Atividades passadas, presentes e futuras, a satisfação com o futuro - ou seja, com o que pode esperar daqui para a frente - foi a faceta com maior correlação ( $r=$ 0,720). Já na Participação social, a faceta de maior relevância foi a que se refere à satisfação com o nível de atividade que desempenha $(r=0,825)$. No domínio Morte e morrer, a faceta de maior impacto foi a que se refere ao medo de não poder controlar a morte $(r=0,856)$. A Intimidade foi influenciada pelas oportunidades para amar $(r=0,951)$.

\section{DISCUSSÃO}

A média de idade dos idosos estudados em Portugal é superior a de outros estudos que utilizaram os instrumentos de QV, indicando ser um grupo mais longevo ${ }^{(12-14)}$.

O estudo com idosos de área adstrita a uma Unidade de Saúde no Brasil ${ }^{(13)}$, que utilizou os mesmos instrumentos de coleta de dados, identificou médias superiores às de Portugal nos escores de qualidade de vida medidos pelo Whoqol-Bref. Em outro estudo, com idosos canadenses e brasileiros ${ }^{(12)}$, igualmente foram observados escores superiores aos idosos de Portugal na QV Global. Na QV Geral, chama a atenção que o grupo português mantenha média inferior a de um grupo de idosos incontinentes, em Porto Alegre ${ }^{(14)}$. Tais diferenças poderiam indicar uma percepção de qualidade de vida inferior dos idosos portugueses em relação a outros grupos.

Tanto entre os idosos portugueses quanto nos idosos do estudo de Creutzberg, Santos e Lovera ${ }^{(13)}$ e dos brasileiros do estudo de Paskulin e Molzahn ${ }^{(12)}$, o domínio que apresentou melhor média foi o das Relações pessoais. Isso poderia indicar que as redes sociais atendem de forma satisfatória às necessidades dos idosos portugueses. Infere-se que a possibilidade de estar em companhia de outros idosos no centro-dia também pode ter influência positiva sobre esse aspecto. Tal referência obteve reforço no relato informal dos idosos portugueses, que expressaram o medo de se sentir só, em função do afastamento de seu grupo familiar, porém preenchiam esse "vazio" com os colegas do centro-dia, encontrando atenção e evitando o sentimento de solidão.

O domínio do Whoqol-Bref que apresentou médias inferiores - e, portanto, menor satisfação para o grupo do presente estudo - foi o Físico. Ressalta-se que o impacto maior no domínio foi o relato da incapacidade para o trabalho, juntamente com a insatisfação com a locomoção. Assim, os idosos portugueses parecem relacionar sua qualidade de vida ao aspecto físico, à possibilidade de manter-se ou não ativo e, por perceberem-se com dificuldades de locomoção, também acabam por diminuir a capacidade para o trabalho. Tal aspecto também pode estar relacionado à longevidade do grupo português. Ainda entre os aspectos mais relevantes para os idosos participantes estão o sentido de vida, as relações sociais e o acesso ao serviço de saúde.

Considerando os escores médios obtidos através do Whoqol-Old, no escore de QV Global-Old, os idosos de Portugal 
obtiveram escore mais elevado do que idosos de estudo realizado em São Paulo(15) e do grupo de idosos não-saudáveis de Fleck, Chachamovich e Trentini ${ }^{(9)}$. A percepção da qualidade de vida foi inferior ao grupo saudável do estudo base de desenvolvimento do Whoqol-Old ${ }^{(9)}$.

Identificou-se que os idosos portugueses demonstraram escores maiores nos domínios Autonomia e Funcionamento do sensório. $\mathrm{O}$ escore alto no domínio Autonomia é interessante do ponto de vista da gerontologia. Nenhum dos grupos estudados por Fleck, Chachamovich e Trentini ${ }^{(9)}$ apresentou destaque nesse domínio. A autonomia é aspecto fundamental a ser mantido no envelhecimento e, segundo estudos, a capacidade do idoso para a tomada de decisão é um construto imprescindível no processo de envelhecimento com qualidade de vida ${ }^{(16)}$. Essa manutenção está, provavelmente, relacionada a aspectos culturais, sendo preservada quando há a valorização e o respeito à capacidade do idoso. Portanto, sendo um dos domínios que se destacam apenas no grupo dos portugueses, infere-se que nesse meio há maior consideração e respeito à autonomia do idoso, gerando mais satisfação nesse aspecto. Os idosos portugueses parecem não sentir sua qualidade de vida afetada tão fortemente pela perda de sentidos, seja na realização de atividades, seja na interação com outras pessoas. Tal sentimento certamente expressa um avanço na inclusão das pessoas, mesmo quando há perdas sensoriais, além do acesso facilitado às tecnologias que garantem sua integração.

A média mais baixa - e, portanto, representando a maior insatisfação dos portugueses - ficou por conta do domínio Atividades passadas, presentes e futuras, sendo que as médias mais baixas foram nas variáveis relacionadas às atividades e oportunidades futuras. Talvez por se tratar de grupo mais idoso do que os demais estudos analisados, a preocupação com o futuro seja mais evidente.

\section{CONCLUSÕES}

A avaliação da QV pelos idosos do estudo demonstra que os centros-dia têm importante papel na manutenção da socialização desse grupo. Também indica a necessidade de garantir o acesso aos serviços de saúde e de que as políticas se pautem na manutenção da capacidade para as atividades da vida diária. Os idosos demonstram que são respeitados em sua autonomia, mas vislumbram poucas oportunidades de satisfação futura, no que os serviços sociais e de saúde podem ter importante influência.

Quanto às diferenças observadas com idosos de outros países, infere-se que aspectos culturais podem influenciar na avaliação da QV, positiva ou negativamente. Expectativas maiores em relação à $\mathrm{QV}$ na velhice podem também gerar uma percepção menos conformista em idosos de países com história mais longa de envelhecimento populacional.

\section{Referências}

1. Instituto Nacional de Estatística (PT). População residente. Lisboa: INE; 2009. 2. Belasco AGS, Sesso RCC. Qualidade de vida: princípios, focos de estudo e intervençōes. In: Diniz DP, Schor N. Qualidade de vida. São Paulo: Manole; 2006. p.1-10.

3. Trentini CM, Chachamovich E, Figueiredo M, Hirakata VN, Fleck MPA. A percepção de qualidade de vida do idoso avaliada por si próprio e pelo cuidador. Rev Estud Psicol. 2006;11(2):191-7.

4. Gonçalves LHT, Alvarez AM. O cuidado na enfermagem gerontogeriátrica: conceito e prática. In: Freitas EV, Py L, Neri AL. Tratado de geriatria e gerontologia. Rio de Janeiro: Guanabara Koogan; 2002. p. 756-61. 5. Diniz DP, Schor N. Guia de qualidade de vida. São Paulo: Manole; 2006. 6. Medronho RA. Epidemiologia. São Paulo: Atheneu; 2003.

7. Bernardino C. Obra Diocesana de Promoção Social - 40 anos. Porto: Claret; 2007.

8. Fleck MPA, Louzada S, Xavier M, Chachamovich E, Vieira G, Santos L, et al. Aplicação da versão em português do instrumento abreviado de avaliação da qualidade de vida Whoqol-Bref. Rev Saúde Pública. 2000;34(2):178-83. 9. Fleck M P, Chachamovich E, Trentini. Development and validation of the Portuguese version of the Wholqol-Old module. Rev Saúde Pública. 2006;40(5):785-91.

10. Duarte OS, Ciconelli RM. Instrumentos para a avaliação da qualidade de vida: genéricos e específicos. In: Diniz DP, Schor N. Qualidade de vida. São Paulo: Manole; 2006. p.11-8.

11. Instituto Brasileiro de Geografia e Estatística. Pesquisa mensal de emprego. O mercado de trabalho segundo cor e raça [Internet]. Disponível em http:// www.ibge.gov.br.

12. Paskulin LM, Molzahn A. Quality of life of older adults in Canada and Brazil [Internet]. Disponivel em http://wjn.sagepub.com/cgi/content/ abstract/29/1/10.

13. Creutzberg M, Santos BRL, Lovera JR. Autocuidado, nível de dependência e qualidade de vida de idosos do distrito leste de Porto Alegre [relatório de pesquisa]. Porto Alegre: PUCRS, Faenfi; 2009.

14. Knorst MR. A influência da intervenção fisioterapêutica sobre a qualidade de vida em pacientes com incontinência urinaria [tese]. Porto Alegre: PUCRS, IGG; 2009 .

15. Figueira HA, Giani TS, Beresford H, Ferreira MA, Mello D, Figueira AA et al. Quality of life (QOL) axiological profile of the elderly population served by the Family Health Program (FHP) in Brazil. Arch Gerontol Geriatr. 2008;49(2009):368-72.

16. Celich K, Creutzberg LHT, Gomes I, Goldim JR. Capacidade para a tomada de decisão em idosos participantes de grupos de convivência para a terceira idade no município de Erechim/RS [tese]. Porto Alegre: PUCRS, IGG; 2009. 